\title{
'|||||||||||||||||||||||||||||||||||||||||||||||||||||||||||||||||||.
}

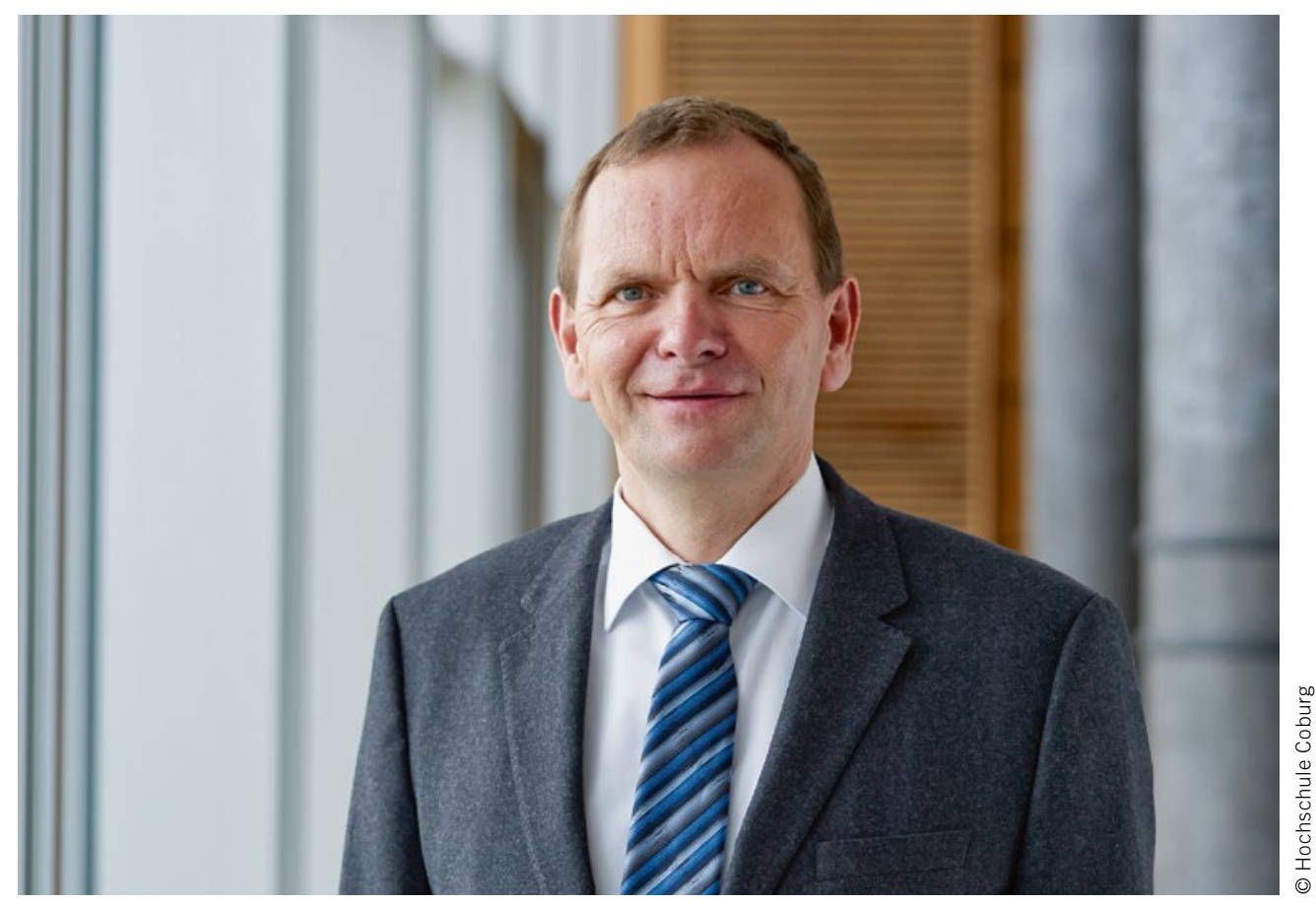

Prof. Dr. Jürgen Krahl

Vorstandssprecher des Technologietransferzentrums Automotive der Hochschule Coburg (TAC)

\section{Im Dreiklang}

Seit Erfindung des Verbrennungsmotors liegt der motorische Forschungsschwerpunkt in einer möglichst effizienten Wandlung von chemischer Energie in Bewegungsenergie. Mit der Einführung der ersten Abgasnormen in den 1960er-Jahren wurde erkannt, dass die Kraftstoffzusammensetzung einen erheblichen Einfluss auf die Verbrennung und damit auf die Emissionen hat. In den 1980er-Jahren wurde Tetraethylblei durch Methyltertiärbutylether (MTBE) ersetzt, um den negativen Auswirkungen von Bleiverbindungen auf die Umwelt entgegenzuwirken.

Aus heutiger Sicht kann die Einführung von bleifreiem Benzin als ein frühes Beispiel des Zusammenspiels von Motortechnik und Chemie angesehen werden. Seit den 1990er-Jahren erfolgte durch den Druck der sich verschärfenden Abgasgesetzgebung die Minderung von Aromaten und Schwefelverbindungen im Kraftstoff. Dieser Schritt war unter anderem erforderlich, um die Langzeitfunktionalität von Abgasnachbehandlungssystemen zu verbessern. Im Vergleich zur rapiden Weiterentwicklung der Motoren und der Abgasnachbehandlung innerhalb der vergangenen 20 Jahre hat sich der Kraftstoff bislang nur moderat geändert. Es darf daher angenommen werden, dass er sein Potenzial im Dreiklang Kraftstoff - Motor - Abgasnachbehandlung noch nicht vollumfänglich entfaltet hat.

Aktuell fokussiert sich die Diskussion darauf, die $\mathrm{CO}_{2}$-Vorgaben zu erfüllen. Der Dieselmotor steht zusätzlich wegen seiner Stickoxidemissionen im öffentlichen und technischen Fokus. Einerseits kann durch Beimischung von Biokomponenten wie
Biodiesel oder hydriertem Pflanzenöl (Hydrotreated Vegetable Oil, HVO) die Klimagasbilanz verbessert werden. Andererseits wurden bei HVO und insbesondere bei Biodiesel auch erhöhte Stickoxidemissionen beobachtet. $\mathrm{Zu}$ diesem Themenkreis besteht mittelfristig Forschungsbedarf, der insbesondere vor dem Hintergrund der zu erwartenden globalen Zunahme der Ressourcendiversifizierung und der gleichzeitigen Forderung nach Dropin-Fuels immer bedeutender wird. $\mathrm{Zu}$ diesen zählen alternative Kraftstoffe, die die konventionellen ohne technische Änderungen ersetzen können und mit ihnen uneingeschränkt mischbar sind.

Eine analoge Situation besteht bei direkteinspritzenden Ottomotoren, deren Neigung zur Partikelbildung bekannt ist. Durch simultane Kraftstoff- und Verbrennungsoptimierung besteht die grundsätzliche Möglichkeit, die Abgasnormen auch ohne Ottopartikelfilter einzuhalten.

Der Kraftstoff ist damit schon heute eine motorische Schlüsselkomponente, die nur im Einklang mit dem Motor und der Abgasnachbehandlung erfolgreich optimiert werden kann.

Die Betrachtung des Kraftstoffs als Energieträger muss daher neben der Untersuchung der Schadstoffbildung um den Ansatz erweitert werden, dass sowohl Kraftstoffe als auch Abgaskomponenten Chemikalien sind, die miteinander reagieren und erwünschte oder unerwünschte Effekte auslösen können. Die Kraftstoffchemie entwickelt den Zweiklang aus motorischer Verbrennung und Abgasnachbehandlung im Sinne einer Parametererweiterung zum Dreiklang. 